\section{IN BRIEF}

\section{SPONDYLOARTHRITIS}

\section{Ixekizumab superior to adalimumab for PsA}

Targeting IL-17A with ixekizumab was superior to targeting TNF with adalimumab in a head-to-head trial involving 566 biologic DMARD-naive patients with psoriatic arthritis (PsA). All patients enrolled had active skin and joint disease and had previously responded poorly to conventional synthetic DMARDs. At 24 weeks, $36 \%$ of patients receiving ixekizumab had reached the primary end point compared with $28 \%$ of those receiving adalimumab. Patients receiving ixekizumab also had fewer serious adverse events than those receiving adalimumab.

ORIGINAL ARTICLE Mease, P. J. et al. A head-to-head comparison of the efficacy and safety of ixekizumab and adalimumab in biological-naïve patients with active psoriatic arthritis: 24-week results of a randomised, open-label, blinded-assessor trial. Ann. Rheum. Dis. https:// doi.org/10.1136/annrheumdis-2019-215386(2019)

\section{AUTOIMMUNITY}

\section{Increased risk of rheumatic disease in IPAF}

Patients with interstitial pneumonia with autoimmune features (IPAF) have a 14-fold higher risk of progressing to a rheumatic disease than patients with interstitial lung disease (ILD) but not IPAF, according to the results of a retrospective cohort study. The term IPAF is used to describe individuals with ILD who have some features of autoimmunity, but who do not fulfil the classification criteria for an autoimmune rheumatic disease. Of the 174 patients included in the study, 8 out of 50 patients with IPAF (16\%) developed rheumatic diseases after a median of 5.2 years, compared with 2 out of 124 patients with ILD (1.6\%). ORIGINAL ARTICLE Alevizos, M. K. et al. Risk of progression of interstitial pneumonia with autoimmune features to a systemic autoimmune rheumatic disease. Rheumatology https://doi.org/10.1093/rheumatology/kez404 (2019)

\section{MYOSITIS}

\section{Combined immunosuppression for ILD in myositis}

A combined immunosuppressive treatment regimen of high-dose glucocorticoids, tacrolimus and intravenous cyclophosphamide was effective in a Japanese cohort of 29 patients with anti-melanoma differentiation-associated gene $5(\mathrm{MDA} 5)^{+}$dermatomyositis-associated interstitial lung disease (ILD) in a prospective study. The 6-month survival of patients receiving combined immunosuppression was higher than that of a historical cohort of 15 patients who received step-up therapy of high-dose glucocorticoids with additional immunosuppressants (89\% versus 33\%, respectively).

ORIGINAL ARTICLE Tsuji, H. et al. A multicenter prospective study of the efficacy and safety of combined immunosuppressive therapy with high-dose glucocorticoid, tacrolimus, and cyclophosphamide in interstitial lung diseases accompanied by anti-melanoma differentiation-associated gene 5-positive dermatomyositis. Arthritis Rheumatol. https://doi.org/10.1002/art.41105 (2019)

\section{THERAPY}

\section{Checkpoint inhibitor-induced arthritis is persistent}

Inflammatory arthritis that developed following immune checkpoint inhibitor (ICl) therapy for cancer persisted for several months following cessation of $\mathrm{ICI}$ therapy and necessitated management by a rheumatologist. At 6 months follow-up, 20 out of 41 patients for whom data were available had active inflammatory arthritis. Overall, three-quarters of the 60 patients included in the follow-up study required immunosuppressive treatment for their inflammatory arthritis, which did not seem to affect cancer progression.

ORIGINAL ARTICLE Braaten, T. J. et al. Immune checkpoint inhibitor-induced inflammatory arthritis persists after immunotherapy cessation. Ann. Rheum. Dis. https://doi.org/10.1136/ annrheumdis-2019-216109 (2019)

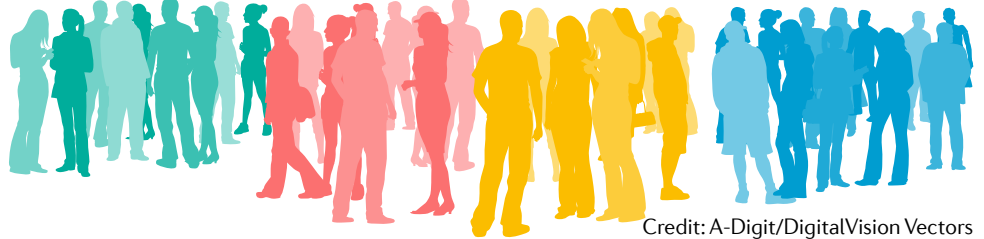

SJÖGREN SYNDROME

\title{
Stratifying Sjögren syndrome into symptom-based subgroups
}

In a new study, symptom-based stratification of patients with primary Sjögren syndrome (pSS) revealed the existence of endotypes that differ with respect to their clinical and biological characteristics and response to therapy. "To our knowledge, this is the first report showing distinct subsets of an immune-mediated inflammatory disease and linking clinical and pathobiological heterogeneity, with direct clinical implications," reports corresponding author Wan-Fai Ng.

Heterogeneity in the clinical presentation of pSS presents challenges in the design of new treatments and in their evaluation in clinical trials. Understanding the differences between patient subgroups could influence pSS management.

In the study, a team of biostatisticians, bioinformaticians, data scientists and clinicians first undertook exploratory clustering analysis of symptom scores for pain, fatigue, dryness, anxiety and depression reported by 608 patients in the UK Primary Sjögren's Syndrome Registry (UKPSSR). The analysis identified four distinct subgroups: low symptom burden, high symptom burden (HSB), dryness dominant with fatigue (DDF) and pain dominant with fatigue. A multinomial logistic regression model was then used to develop a tool to stratify other patients with pSS into these four symptom-based subgroups.

Comparison of the subgroups in the UKPSSR cohort revealed substantial differences in salivary flow, ocular dryness, serum IgG concentrations, peripheral blood lymphocyte counts and prevalence of anti-SSA or anti-SSB antibodies; whole-blood transcriptomic profiles also varied across the subgroups. These differences were also observed in two independent validation cohorts of patients in Norway $(n=62)$ and France $(n=334)$.

"A vital lesson that we have learned is the importance of collecting patient reported outcomes, not only because they matter to the patients, but also because they help us to do better research," Ng says.

Application of the symptombased stratification scheme in a reanalysis of data from two placebocontrolled phase III trials in pSS showed that treatment response varied across the subgroups. In the TRACTISS trial, patients in the DDF subgroup showed improved salivary flow in response to rituximab treatment, and in the JOQUER trial, patients in the HSB subgroup showed improvement in symptoms in response to hydroxychloroquine treatment, whereas no treatment effect was seen in the other subgroups.

"We believe that our findings have key implications for drug development, particularly in clinical trial design, as well as informing molecular targets," says Ng. "Knowledge of these subtypes will also help us to develop a more personalised management plan for individual patients."

Sarah Onuora

ORIGINAL ARTICLE Tarn, J. R. et al. Symptom-based stratification of patients with primary Sjögren's syndrome: multi-dimensional characterisation of international observational cohorts and reanalyses of randomised clinical trials. Lancet Rheumatol. 1, e85-94 (2019) 